\title{
Climate change and the future health of children in low-income countries
}

Sara Ajanovic ${ }^{1}$, Marta Valente ${ }^{1}$, Rosauro Varo ${ }^{1}$, Quique Bassat ${ }^{1,2,3,4,5}$

1. ISGlobal, Hospital Clínic - Universitat de Barcelona, Barcelona, Spain

2. Centro de Investigação em Saúde de Manhiça (CISM), Maputo, Mozambique

3. ICREA, Pg. Lluís Companys 23, 08010 Barcelona, Spain.

4. Pediatric Infectious Diseases Unit, Pediatrics Department, Hospital Sant Joan de Déu (University of Barcelona), Barcelona, Spain

5. Consorcio de Investigación Biomédica en Red de Epidemiología y Salud Pública (CIBERESP), Madrid, Spain

As paediatricians, working globally, we have a professional and moral responsibility to guarantee the health of those who are children today, but also of the children born in the years to come. The climate crisis, perhaps the biggest threat to human survival in the foreseeable future, will disproportionately affect the health of children, and particularly of those born in resource constrained settings. We urgently need to start taking the necessary steps to adequately predict and thus prevent its impact on the health of children.

\section{Climate change}

Climate change could be defined as the long-term variation of temperature and typical weather patterns in a concrete area (or the whole planet). Climate change will likely have a negative impact on environmental and social determinants of health. The accelerated pace of climate change allows us to define it as an emergency which calls for immediate responses. "Climate action" is indeed the $13^{\text {th }}$ Sustainable Development Goal. According to the United Nations Development Program 2018 report (1), the world has experienced a 1 으 $\mathrm{C}$ temperature rise above pre-industrial levels; and sea levels have risen about $20 \mathrm{~cm}$ since 1880 . The world is currently witnessing severe floods, storms, heatwaves, and droughts, as well as unpredictable outbreaks of infectious and noninfectious diseases, often affecting a variety of geographical settings where they had seldom occurred (2-5). Considering this evidence, there is a spectrum of possible future scenarios depending on the degree of commitment and action we are willing to take. The Paris Agreement has targeted a $2^{\circ} \mathrm{C}$ limit above pre-industrial levels in order to reduce the effects of climate change on human health. According to climate experts, this is a two-pathway decision: either continue with the "usual business" response or completely redirect the efforts towards a "well below $2{ }^{\circ} \mathrm{C}$ " future. A recent Lancet report suggests that we are taking on the former rather than on the latter: "the world is following this "business as usual" pathway and it will lead to a profoundly altered world in which the life of every child born today will be substantially affected" (6).

\section{Age inequities}

Climate change will disproportionally hit children. Children under five years of age are estimated to suffer $88 \%$ of the disease burden attributable to climate change in terms of disability-adjusted life years (7). Many reasons contribute to this, including the immature physiology and baseline metabolism; the "windows of vulnerability" during their in utero life and early childhood; the higher exposure per unit body weight (as they 
breathe more air, drink more water and eat more food proportionally); and their unique diet and behavioural patterns. Furthermore, they are not fully independent and their ability to face environmental hazards depends on their caregiver's and local community's resources. (8).

\section{Geographical inequities}

The effects of climate change will not be equally distributed geographically and will contribute to increasing inequities in resources and wealth between countries and regions. Although low-income countries (LICS) produce less than $10 \%$ of global greenhouse gas emissions, they remain the most vulnerable to the effects of climate change. This could likely reverse many of the accomplishments achieved in the last 50 years in terms of health inequalities and push back one third of the population to extreme poverty by 2050 (9).

\section{Climate health impact in the health of children from LIC}

Examples of this include the impact on child health of droughts and floods, which will affect agricultural yields in areas already experiencing food insecurity, potentially leading to a consequent rise in malnutrition. These may also contribute to water and food contamination and compromise the already difficult access to safe water and improved sanitation in these regions. Similarly, extreme heat waves will likely hit harder those who don't have resources to face them, with children being naturally more vulnerable. Large population displacements will also affect health, economic development and political instability further jeopardizing the health of the affected people who have less tools to face those hazards (8). During massive displacements or post-disaster situations, outbreaks of infectious diseases, violent conflicts over scarce resources and continuous food insecurity will consequently arise. In these scenarios, children will pay the highest price, as their lives will be the most exposed and disrupted (7). Environmental and social conditions also make these children more exposed to infectious disease agents since they spend more time outdoors, being in more contact with microorganisms and vectors, and are more likely to eat and drink from contaminated sources (10). The distribution of the world's 2.3 billion children is important to understand where the consequences will be greater. In 2015 UNICEF warned that "over half a billion children were living in areas with extremely high levels of flood occurrence, and nearly 160 million lived in areas of high or extremely high drought severity". Most of these areas were located in Africa and Asia (11). Population growth is disproportionately affecting low-income regions, where the majority of the new children are being born. Indeed, UNICEF statistics predict that by 2050 more than a third of the children in the world will live in sub-Saharan Africa (12). Considering that these regions are already suffering the brunt of poverty-related health issues, it is foreseeable that these will only get exacerbated as a result of climate change.

In summary, children born today in LICs are more likely to endure the adverse health consequences of climate change and are doubly affected, both by geographical and age inequities, perpetuating the intergenerational cycle of poverty $(13,14)$. 


\section{Child mortality}

Climate change is also estimated to have a significant impact on child mortality. According to the World Health Organization in 2018 an estimated 6.2 million children and adolescents under 15 years died, mostly from preventable causes. Eighty-five percent of these deaths occurred in the first 5 years of life, and nearly half of those in the first month of life. Leading causes of death in children under 5 years of age include neonatal deaths, acute respiratory infections, diarrhoea, and malaria (9). The most important actions in order to prevent those deaths are immunization, adequate nutrition, safe water and food and appropriate care by a trained health provider when necessary. While children mortality has halved in the last 25 years (15), some evidence suggests it may start increasing again as a result of climate change. A recent review on heat exposure and maternal health (16), showed the impact of heat on birth outcomes leading to an increase on preterm birth, low birth weight and stillbirth rates (16). Regarding respiratory diseases, temperature variability seems to increase the number of pneumonia cases and exacerbate the effects of allergens and air pollution(17-20), and diarrheal cases could also increase. There is uncertainty on the direct impact of climate change in terms of malaria burden, but there is good evidence to suggest that vector-borne diseases in general will increase (21). Importantly, the number of people living in malaria transmission areas will increase to $60 \%$ by 2100 (9). In relation to undernutrition, its prevalence will increase by $16 \%$ due to poorer access to food, affecting in a cross-cutting way all the other death causes too. Children living through large populational displacements and major climate disasters, will have poorer access to preventive measures, such as vaccination, adequate sanitation and hygiene. Hence, both indirect risk factors and direct child death causes will increase.

\section{Conclusions}

The depiction of such an apocalyptic future scenario is not gratuitous. Climate change is a threat to many of the gains slowly and painfully achieved in child health and mortality prevention, and the Global Health community needs to commit and prioritize the fields on which to focus. As paediatricians working in global child health, we need to be at the forefront of the efforts to anticipate, prevent and tackle future child health problems derived from our own action. Indeed, "No human responsibility runs deeper than the charge of every generation to care for the generation that follows it" (11).

\section{Bibliographical References}

1. Sangani K. The heat is on. Eng Technol. 2014;9(7):49-51.

2. Semenza JC, Suk JE. Vector-borne diseases and climate change: A European perspective. FEMS Microbiol Lett. 2018;365(2):1-9.

3. Zakham F, Alaloui A, Levanov L, Vapalahtil O. Viral haemorrhagic fevers in the Middle East. J Chem Inf Model. 2019;53(9):1689-99.

4. Johnson RJ, Wesseling C, Newman LS. Chronic kidney disease of unknown cause in agricultural communities. N Engl J Med. 2019;380(19):1843-52.

5. Bloom DE, Cadarette D. Infectious disease threats in the twenty-first century: Strengthening the global response. Front Immunol. 2019;10(MAR).

6. Watts N, Amann M, Arnell N, Ayeb-Karlsson S, Belesova K, Boykoff M, et al. The 
2019 report of The Lancet Countdown on health and climate change: ensuring that the health of a child born today is not defined by a changing climate.

Lancet. 2019;394(10211):1836-78.

7. Zhang $\mathrm{Y}, \mathrm{Bi} \mathrm{P}, \mathrm{JE}$ H. Climate change and disability -- adjusted life years.

[Internet]. Vol. 70, Journal of Environmental Health. 2007. p. 32-6. Available from:

http://proxy.library.adelaide.edu.au/login?url=http://search.ebscohost.com/log

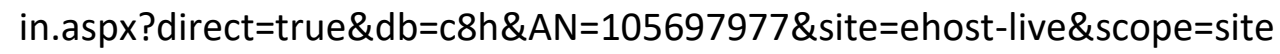

8. Sheffield PE, Landrigan PJ. Global climate change and children's health: Threats and strategies for prevention. Environ Health Perspect. 2011;119(3):291-8.

9. Kiang K, Graham S, Farrant B. Climate change, child health and the role of the paediatric profession in under-resourced settings. Trop Med Int Heal.

2013;18(9):1053-6.

10. Stanberry LR, Thomson MC, James W. Prioritizing the needs of children in a changing climate. PLoS Med. 2018;15(7):11-4.

11. UNICEF. Unless we act now. 2015.

12. UNICEF analysis based on the United Nations, Department of Economic and Social Affairs, Population Division, World Population Prospects: The 2015 Revision, United Nations, New York, 2015.

13. Philipsborn RP, Chan K. Climate change and global child health. Pediatrics. 2018;141(6).

14. Bennett C, Friel S. Impacts of Climate Change on Inequities in Child Health. Children. 2014;1(3):461-73.

15. You D, Hug L, Ejdemyr S, Idele P, Hogan D, Mathers C, et al. Global, regional, and national levels and trends in under-5 mortality between 1990 and 2015, with scenario-based projections to 2030: A systematic analysis by the un InterAgency Group for Child Mortality Estimation. Lancet [Internet]. 2015;386(10010):2275-86. Available from: http://dx.doi.org/10.1016/S01406736(15)00120-8

16. Kuehn L, McCormick S. Heat exposure and maternal health in the face of climate change. Int J Environ Res Public Health. 2017;14(8).

17. Sangho Sohn et al. 'Pneumonia Weather': Short-term Effects of Meteorological Factors on Emergency Room Visits Due to Pneumonia in Seoul, Korea. J Prev Med Public Health 2019;52:82-91.

18. Sun S, Laden F, Hart JE, Qiu H, Wang Y, Wong CM, et al. Seasonal temperature variability and emergency hospital admissions for respiratory diseases: A population-based cohort study. Thorax. 2018;73(10):951-8.

19. $\mathrm{Xu} \mathrm{Z,} \mathrm{Hu} \mathrm{W,} \mathrm{Tong} \mathrm{S.} \mathrm{Temperature} \mathrm{variability} \mathrm{and} \mathrm{childhood} \mathrm{pneumonia:} \mathrm{An}$ ecological study. Environ Heal A Glob Access Sci Source. 2014;13(1):1-8.

20. Mehdi Mirsaeidi et al. Climate Change and Respiratory Infections. Ann Am Thorac Soc. 2016 Aug;13(8):1223-30.

21. Parham PE et al. 2015 Climate, environmental and socio-economic change: weighing up the balance in vector-borne disease transmission. Phil. Trans. R. Soc. B 370: 20130551. 